\title{
Health maintenance tables and completion of cervical cancer screening: a cross-sectional observational study
}

\author{
Suellen A Romero de Mello Sa ${ }^{1}$, Lazarus K Mramba ${ }^{2}$ and Maryam Sattari ${ }^{3 *}$ \\ ${ }^{1}$ University of Florida College of Medicine, Gainesville, FL, USA \\ ${ }^{2}$ Department of Medicine, University of Florida College of Medicine, Gainesville, FL, USA \\ ${ }^{3}$ Division of General Internal Medicine, Department of Medicine, University of Florida College of Medicine, Gainesville, FL, USA
}

\begin{abstract}
Significant gaps exist in evidence-based screening for cervical cancer. Effective interventions to improve screening rates are essential to bridge this gap and reduce morbidity and mortality. To improve screening, we created a provider reminder tool called the health maintenance table (HMT). The aim of this study was to investigate the association of HMT use with cervical cancer screening. We hypothesized that HMT utilization in the documentation by primary care providers (PCPs) is associated with higher completion of evidence-based cervical cancer screening.

The University of Florida (UF) IRB approved this retrospective study. We included female patients 21 to 65 years of age without a history of cervical cancer or hysterectomy who visited the internal medicine clinic at the Medical Plaza at UF between 05/31/2016-06/21/2016. PCPs in the clinic had the option of using the HMT, a computer-generated reminder .HM, a typed annotation of preventive care, or nothing at all. Analysis, based on logistic regression models, was completed in 2016.

HMT use was strongly associated with completing cervical cancer screening compared to HMT non-use, $(\mathrm{OR}=4.18,95 \% \mathrm{CI}=[1.723,10.144]$, p-value = 0.002), after adjusting for potential confounding variables and risk factors in the multiple logistic regression model.

Our findings suggest that HMTs may provide a sustainable model for improving completion of evidence-based cervical cancer screening. The HMT has several benefits, including being adaptable to fit each patient and placed within a visit note as both a reminder and documentation for all steps involved in offering preventive care measures.
\end{abstract}

\section{Introduction}

The American Cancer Society estimates that 12,990 women will be diagnosed with invasive cervical cancer and 4,120 women will die from the disease in 2016 [1]. Although cervical cancer incidence and cancer-related death rates have decreased since the implementation of Papanicolaou (pap) smears, cervical cancer remains a public health concern owing to significant gaps in routine screening [2]. According to the Centers for Disease Control and Prevention, only $70 \%$ of women are receiving appropriate screening [3]. Furthermore, socioeconomic disparities exist in cervical cancer screening rates that result in differences in incidence of cancer as well as associated morbidity and mortality. For example, screening rates are 25\% higher in insured (85.6\%) compared with uninsured women (60.6\%) [4]. Importantly, morbidity and mortality from cervical cancer correlate with screening. Over $50 \%$ of women diagnosed with cervical cancer have either never had a pap smear or not received one in the last five years [5]. An additional $10 \%$ of women never returned to follow-up on abnormal findings [5]. In 2012, screening guidelines were updated to include human papillomavirus (HPV) DNA co-testing with cytology for women 30 to 65 years of age [6]. Cervical cancer screening can reduce cancer-related morbidity and mortality by detecting and treating precancerous and cancerous lesions early [7].

Effective interventions to improve screening rates are essential to bridge the screening gap. Patient reminders, such as letters mailed to patients near the time they are due for screening, aid in increasing completion of several preventive care procedures [8,9]. Clinician reminders in various formats (paper-based, computer-generated, or a combination thereof) have also demonstrated efficacy in improving the completion of preventive care services [10-12]. Paper-based clinician reminders include memos, stickers, or a slip of paper on the patient's chart to remind the providers. Computerized reminders use algorithms to determine eligible patients and provide prompts upon access to the clinical information system. In a systematic review, Dexheimer and colleagues reported a $12-14 \%$ average increase in delivering preventive care measures for physicians who utilize reminder systems [10].

Since existing studies have demonstrated only moderate correlation between clinician reminders and compliance with evidence-based guidelines $[11,13,14]$, we decided to develop a provider-created reminder tool for evidence-based preventive care at the University of Florida (UF) College of Medicine (Gainesville, Florida). Our goal was to create a tool that addresses all evidence-based preventive care

*Correspondence to: Maryam Sattari, Division of General Internal Medicine, University of Florida College of Medicine, PO Box 103204, 1329 SW 16th Street, Suite 5140, Gainesville, FL 32610-0277, USA, Tel: (352) 265-0651; Fax: (352) 265-0153; E-mail: maryam.sattari@medicine.ufl.edu

Key words: cervical cancer, screening, provider reminder, Pap smear, cytology, $H P V$, health maintenance, preventative care, reminder system

Received: April 28, 2018; Accepted: May 21, 2018; Published: May 25, 2018 
measures, can be adapted to fit each patient, and can be placed within a visit note (either in a paper or electronic chart). We aimed to create a tool to not only remind clinicians about preventive care measures, but also facilitate documentation of all steps involved in offering these measures (indications, dates of service, results, as well as next due dates for each service) within the medical record. We reviewed the literature and incorporated evidence-based guidelines from national medical professional societies and preventive service organizations, including the United States Preventive Services Task Force (USPSTF), to create two gender-specific health maintenance tables (HMTs). The femalespecific HMT consists of 19 items, including screening for cervical cancer (Table 1). HMT use was associated with increased influenza vaccination in adult patients [15]. However, vaccinations are in general easier to perform during the same visit and may be more likely to be completed compared to cervical cancer screening, which usually requires a separate appointment or referral.

The current study aims to investigate the association of HMT use with evidence-based cervical cancer screening. We hypothesized that the use of HMTs in the documentation within the electronic medical records (EMRs) by primary care providers (PCPs) would be associated with higher completion of evidence-based cervical cancer screening. The rationale for this hypothesis is that the HMT is likely to not only remind PCPs of evidence-based preventive care services, but also assist them in easily tracking the services offered, completed, and needing to be completed by their patients.

\section{Methods}

IRB approval: The UF Institutional Review Board (IRB) approved this study

\section{Setting}

HMTs were implemented in one Internal Medicine clinic at UF and subsequently adopted by many of the PCPs (both attending and resident providers) at this clinic for routine use in the EMR. The PCPs are not obligated to utilize the HMT and have the option of using the HMT, the EMR-generated reminder with the smart phase .HM, a simple typed annotation of preventive care, or nothing at all. The smart phrase .HM is a recently-implemented module at UF that contains a

Table 1: Example of a Female Health Maintenance Table (HMT)

\begin{tabular}{|l|l|l|l|}
\hline TEST & Date of Last Test & Comments & Next Test Due \\
\hline Health Exam & & & \\
\hline Breast cancer screening & & & \\
\hline Breast cancer risk & & & \\
\hline Cervical cancer screening & & & \\
\hline Colorectal cancer screening & & & \\
\hline Lung cancer screening & & & \\
\hline ASCVD risk & & \\
\hline DEXA/FRAX score & & \\
\hline Contraception & & \\
\hline Hepatitis C screening & & \\
\hline HIV screening & & \\
\hline Gestational complications & & \\
\hline Varicella/Rubella & & \\
\hline Flu vaccination & & \\
\hline Pneumococcal vaccination & & \\
\hline Tetanus/Tdap vaccination & & \\
\hline Zoster vaccination & & \\
\hline HPV vaccination & & \\
\hline Hepatitis B vaccination & & \\
\hline
\end{tabular}

list of computerized reminders about preventive care procedures, the date most recently completed, and the next due date. Providers can insert this module into their documentation by typing the smart phrase .HM. A retrospective chart review using the EMR Epic was performed for all patients who visited this clinic between the dates 05/31/2016 and $06 / 21 / 2016$

\section{Sample selection and definitions}

We used cervical cancer screening guidelines from the USPSTF to determine eligibility for inclusion in our study [6]. We included female patients 21 to 65 years of age at the time of their clinic visit without a personal history of cervical cancer or hysterectomy [6]. Women ages 21 to 29 years were considered up-to-date on screening if they had received a cytology (pap smear) screening within 36 months of the visit. Women ages 30 to 65 years were considered up-to-date on screening if they had undergone cytology alone within 36 months or cytology and HPV co-testing within 60 months of the visit.

\section{Data sources and measures}

We extracted the following information for eligible patients who had at least one clinic visit within the study dates: (a) the type of physician reminder system included in the clinical encounter documentation, (b) the patient's age at the time of clinic visit, (c) gender, (d) medical insurance, (e) education level, (f) marital status, (g) English proficiency by inferring from the patient's preferred language, (h) whether the regular PCP was a resident or faculty physician, (i) whether the patient was eligible for cervical cancer screening at the time of clinic visit, $(j)$ whether the patient underwent evidence-based cervical cancer screening, and (k) HPV DNA status if available. Screening was considered completed if the patient actually underwent the screening procedure at the internal medicine clinic, another UF clinic, or a nonUF facility, independent of the results. We also collected information about .HM utilization for each visit and determined if the provider made a simple typed out annotation about preventive care services. Patients who visited the clinic more than once during the dates of the study were only included once. The main exposure variable was the type of provider reminder system used while the main outcome of interest was cervical cancer screening completion as defined above. Potential confounders included age, race, marital status, insurance type, and physician level, which were adjusted for in the multiple logistic regression models.

\section{Bias}

Possible sources of bias would have been exposure misclassification if providers reported a different form of reminder system than the one they actually used. Therefore, we obtained information on the true source of reminder directly from medical records. In addition, selection bias would have been introduced if we included only patients at high risk for cervical cancer since they are more likely to undergo screening than healthy average-risk women. Therefore, we included all female patients who satisfied the inclusion criteria in this study.

\section{Statistical methods}

Descriptive statistics, including student's t-test, Chi-square test of independence, and Fisher's exact test were performed. Univariate associations between the response variable (cervical cancer screening completion) and each of the independent variables of interest were analyzed using simple logistic regression models. Akaike Information Criterion (AIC) and residual deviances were used as the diagnostic criteria for choosing the best model. Multiple logistic regression models were fitted to adjust for the association between the outcome and a given 
covariate of interest while holding the other independent variables in the model constant. Analysis was done using Stata statistical software [16]. There were no missing data with respect to the response variable and the exposure variable of interest. The sampling strategy entailed a one-stage simple sampling where all women who satisfied the inclusion criteria were included in the study. Sensitivity and specificity analyses were not applicable since we are not comparing our results to standard results obtained using a gold standard. Since this was a cross-sectional design, there was no attrition.

\section{Results}

\section{Descriptive analysis}

We identified 252 eligible female patients who visited the internal medicine clinic during the study period. The average age of participants was 46 years (standard error $=0.83$ years) at the time of study (Table 1). Most patients ( $\mathrm{n}=106 ; 42.1 \%)$ were married, 98 (38.9\%) single, 37 (14.7\%) divorced, and 11 (4.4\%) had unknown marital status (Table 1). Insurance types were categorized as private or through employer $(\mathrm{n}=$ 154, 61.1\%); Medicaid, Medicare, or Tricare $(\mathrm{n}=85,33.7 \%)$; and none $(\mathrm{n}=13,5.2 \%)$. Three race categories were used: white/Caucasian $(\mathrm{n}=$ 149, 59.1\%), black/African American $(\mathrm{n}=74,29.4 \%)$, and Asian $(\mathrm{n}=$ $5,2.0 \%)$. Twenty-four (9.5\%) participants did not identify their race or reported other, and 20 patients $(7.9 \%)$ reported Hispanic or Latina ethnicity. We found a $71.0 \%$ cervical cancer screening rate. Out of the 179 patients who had completed cervical cancer screening, 139 (77.7\%) also had documented HPV DNA testing (Table 2).

Fifty-seven PCPs documented at least one visit of a study-eligible patient during the study interval. The visits were classified into those done by attending physicians only $(\mathrm{n}=147,58.3 \%)$ and those done primarily by resident physicians ( $\mathrm{n}=105,41.7 \%$ ). HMT use was documented in $83(32.9 \%)$ encounters, .HM smart phrase use in $35(13.9 \%)$, and simple annotations in 38 (15.1\%). The remaining 97 (38.5\%) encounters did not use any of these methods. One of the patient's charts included both HMT and .HM; thus, this patient was included in both reminder system categories.

\section{Univariate (Crude/Un-adjusted) analysis}

HMT use was positively associated with completion of cervical cancer screening in the simple logistic model $(\mathrm{OR}=2.64,95 \% \mathrm{CI}=$ $[1.372,5.093], \mathrm{p}$-value $=0.004)$ (Figure 1a). There was no statistically significant association between cervical cancer screening completion and utilization of the computer-generated reminder with the smart phrase.$H M$ ( $p$-value $=0.124$ ) or a simple annotation ( $p$-value $=0.058$ ). Use of at least one of these reminder systems was associated with increased odds of completing cervical cancer screening ( $\mathrm{OR}=2.39,95 \%$ $\mathrm{CI}=[1.372,4.174], \mathrm{p}$-value $=0.002)$ (Figure $1 \mathrm{~b})$. A univariate model was fitted with a categorical exposure variable with 4 levels: $0=$ HMT use, $1=$. HM use, $2=$ Simple Annotation, and $3=$ None. Compared to HMT use, both. $\mathrm{HM}$ use $(\mathrm{OR}=0.31,95 \% \mathrm{CI}=[0.0127,0.750]$, $\mathrm{p}$-value $=0.009)$ and not using any reminder system $(\mathrm{OR}=0.31,95 \% \mathrm{CI}=$ $[0.151,0.619]$, $\mathrm{p}$-value $=0.001)$ were associated with $69 \%$ reduction in the odds of completing cervical cancer screening (Figure 1c).

Age was not associated with completion of cervical cancer screening $(\mathrm{OR}=1.01,95 \% \mathrm{CI}=[0.986,1.028], \mathrm{p}$-value $=0.526)$. Similarly, the crude associations between the outcome variable and ethnicity, physician level (attending or resident), and insurance type were not
Table 2: Results of descriptive analysis

\begin{tabular}{|c|c|c|}
\hline Patient's Age at the time of Clinic Visit & Frequency & $\%$ \\
\hline $21-30$ & $44 / 252$ & 17.5 \\
\hline $31-40$ & $44 / 252$ & 17.5 \\
\hline $41-50$ & $55 / 252$ & 21.8 \\
\hline $51-60$ & $64 / 252$ & 25.4 \\
\hline $61-65$ & $45 / 252$ & 17.9 \\
\hline \multicolumn{3}{|l|}{ Race } \\
\hline White & $149 / 252$ & 59.1 \\
\hline Black or African American & $74 / 252$ & 29.4 \\
\hline Asian & $5 / 252$ & 2.0 \\
\hline Other/missing & $24 / 252$ & 9.5 \\
\hline \multicolumn{3}{|l|}{ Ethnicity } \\
\hline Hispanic & $20 / 252$ & 7.9 \\
\hline \multicolumn{3}{|l|}{ Preferred Language } \\
\hline English & $245 / 251$ & 97.6 \\
\hline Other & $6 / 251$ & 2.4 \\
\hline \multicolumn{3}{|l|}{ Marital Status } \\
\hline Single & $98 / 252$ & 38.9 \\
\hline Married or Domestic Partnership & $106 / 252$ & 42.1 \\
\hline Divorced, Separated, or Widowed & $37 / 252$ & 14.7 \\
\hline Missing & $11 / 252$ & 4.4 \\
\hline \multicolumn{3}{|l|}{ Educational Level (Years) } \\
\hline Less than High School $(<12)$ & $1 / 55$ & 1.8 \\
\hline High School Diploma or GED (12) & $7 / 55$ & 12.7 \\
\hline Some College, Associate Degree, or Technical School (13-15) & $17 / 55$ & 30.9 \\
\hline Bachelor's Degree (16) & $17 / 55$ & 30.9 \\
\hline Beyond Bachelor's Degree (>16) & $13 / 55$ & 23.6 \\
\hline \multicolumn{3}{|l|}{ Insurance Type } \\
\hline Private or Through Employer & $154 / 252$ & 61.1 \\
\hline Medicaid, Medicare, or Tricare & $85 / 252$ & 33.7 \\
\hline No Insurance Coverage & $13 / 252$ & 5.2 \\
\hline \multicolumn{3}{|l|}{ Provider Type } \\
\hline Attending Physician & $147 / 252$ & 58.3 \\
\hline Resident & $105 / 252$ & 41.7 \\
\hline \multicolumn{3}{|l|}{ Reminder System Utilized* } \\
\hline HMT & $83 / 253$ & 32.9 \\
\hline. $\mathrm{HM}$ & $35 / 253$ & 13.9 \\
\hline Typed out annotation & $38 / 253$ & 15.1 \\
\hline None & $97 / 253$ & 38.5 \\
\hline Completed Cervical Cancer Screening & $179 / 252$ & 71.0 \\
\hline Completed HPV Testing & $139 / 252$ & 55.2 \\
\hline \multicolumn{3}{|l|}{ HPV DNA Status } \\
\hline Negative & $125 / 136$ & 91.9 \\
\hline Positive & $11 / 136$ & 8.1 \\
\hline
\end{tabular}

$\mathrm{HMT}=$ health maintenance table, $. \mathrm{HM}=$ computer generated provider reminder, $\mathrm{HPV}=$ human papillomavirus

* One patient's chart included both HMT and .HM use; thus, this patient was counted in both reminder system categories.

statistically significant. Black race had a non-significant association with the odds of completing cervical cancer screening, compared to white race $(\mathrm{OR}=1.86$, $\mathrm{p}$-value $=0.064)$.

\section{Multiple logistic regression analysis}

Controlling for age, HMT use, insurance type, physician level, and marital status, black/African American race was associated with increased odds of completing cervical cancer screening compared to white/Caucasian race $(\mathrm{OR}=2.37,95 \% \mathrm{CI}=[1.110,5.088]$, $\mathrm{p}$-value $=$ 0.026) (Figure 2a). Being married was associated with a two-fold increase in odds of completing cervical cancer screening compared to being single $(\mathrm{OR}=2.24,95 \% \mathrm{CI}=[1.004,5.010], \mathrm{p}$-value $=0.049)($ Figure 2a $)$. 


\section{Comparison of HMT versus No HMT use}

HMT use had 4.18 times the odds of completing cervical cancer screening than HMT non-use $(\mathrm{OR}=4.18,95 \% \mathrm{CI}=[1.723,10.144]$, p-value $=0.002)($ Figure 2a) .

Comparison of Using at Least One Reminder Method versus No Tracking of Preventive Care

The use of at least one of the health maintenance reminder systems was associated with increased odds of completing cervical cancer screening compared to not using any method of tracking preventive care $(\mathrm{OR}=2.32,95 \% \mathrm{CI}=[1.198,4.485]$, $\mathrm{p}$-value $=$ 0.013) (Figure 2b).

\section{Comparison of .HM versus No.HM use}

.HM use was not associated with the outcome variable, even after adjusting for age, marital status, insurance type, race, and physician type $(\mathrm{OR}=1.011$, $\mathrm{p}$-value $=0.160)$.

\section{Comparison of HMT use versus .HM, Simple Annotation, and No Tracking of Preventive Care}

Controlling for age, marital status, insurance type, race, and physician level in the model, use of .HM was associated with $84 \%$ reduced odds of completing cervical cancer screening compared to HMT use $(\mathrm{OR}=0.16,95 \% \mathrm{CI}=[0.049,0.500], \mathrm{p}$-value $=0.001)$ (Figure 2c). Not using any reminder systems was associated with $81 \%$ reduced odds of cervical cancer screening completion compared to HMT use $(\mathrm{OR}=0.19,95 \% \mathrm{CI}=[0.072,0.488], \mathrm{p}$-value $=0.001)$ (Figure 2c) . While simple annotation was associated with $40 \%$ reduction in odds of completing cervical cancer screening compared to HMT use, the association was not statistically significant $(\mathrm{OR}=0.60,95 \%$, p-value $=0.409)$.

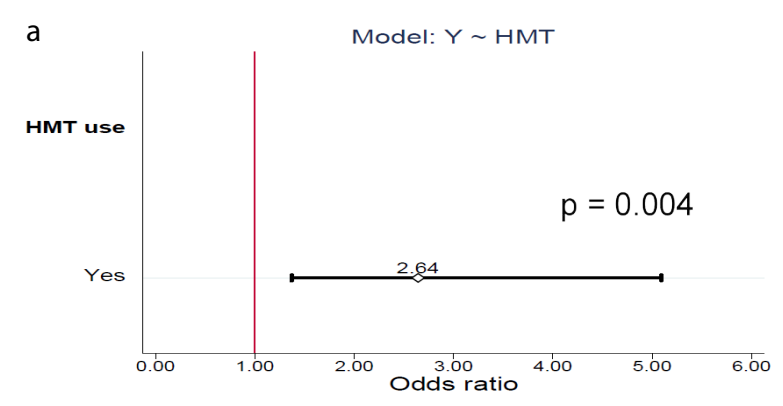

\section{Discussion}

Previous provider reminder systems have been associated with moderate increases in compliance with evidence-based guidelines, but there continues to be a need for innovative, well-designed reminders that better fit with the people, process, and technology of health care delivery $[10,11,13,14]$. The HMT is a novel provider reminder that resulted in high adoption (38\%) compared to previously cited adoption of $10-20 \%[17,18]$. Furthermore, HMT use was associated with significantly higher odds of evidence-based cervical cancer screening, even when we included other reminder modalities (e.g. .HM and typed out annotations) in the non-HMT group and controlled for physician level and the patient's age, race, marital status, and insurance type.

Interestingly, the difference between cervical cancer screening odds for HMT users and providers who typed out a simple annotation about preventive care services (which did not always include cervical cancer screening) was not statistically significant. This finding could reflect the relatively small size of the two groups as well as the importance of provider recognition of the significance of these services, regardless of their actual documentation/tracking method. Potential explanations for the significantly lower completion of screening among .HM users include the small number of physicians in this group, the recent implementation of the module, and "prompting fatigue" of computerized reminder systems resulting in physicians simply ignoring the reminders in order to move on with the visit [10]. Physicians might also glance over computer-generated reminders because they are not consistently relevant to the patient at hand. For instance, our .HM marked patients with a history of hysterectomy for benign reasons as overdue for cervical cancer screening. On the other hand, the HMT might actively engage the providers as they have to manually enter results and decide on next due dates.

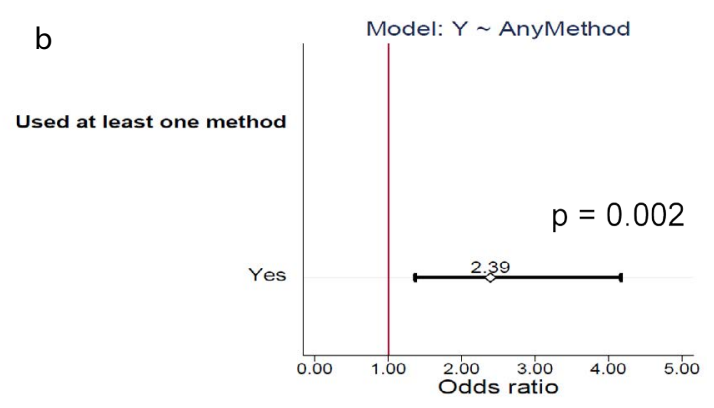

C

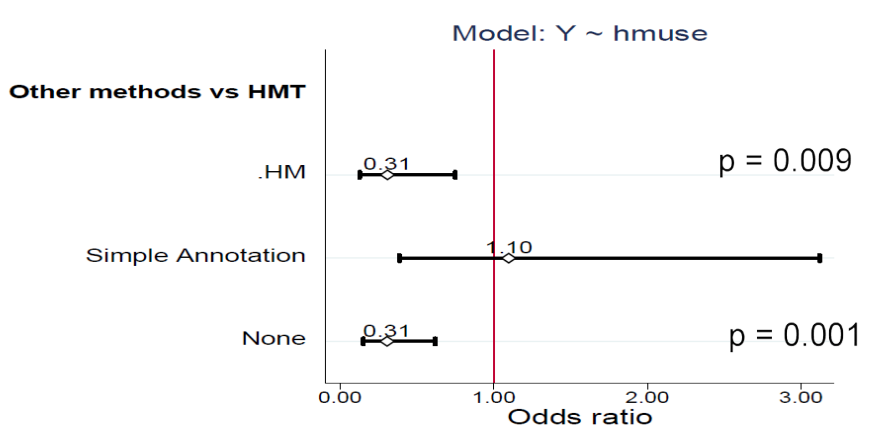

Figure 1: Results of Simple Logistic Regression Model or Univariate (crude/un-adjusted) Analysis. a. HMT use was positively associated with completion of cervical cancer screening. b. Use of at least one reminder system (HMT, .HM, or simple annotation) was associated with increased odds of cervical cancer screening. c. Compared to HMT use, both .HM use and not using any reminder system were associated with $69 \%$ reduction in the odds of cervical cancer screening. 
a

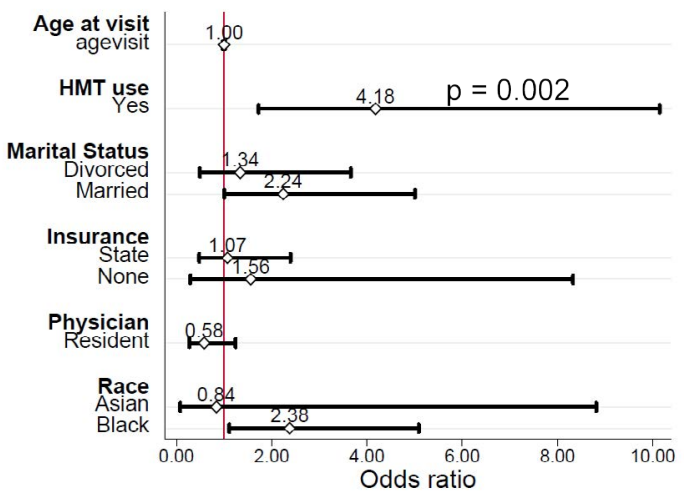

b

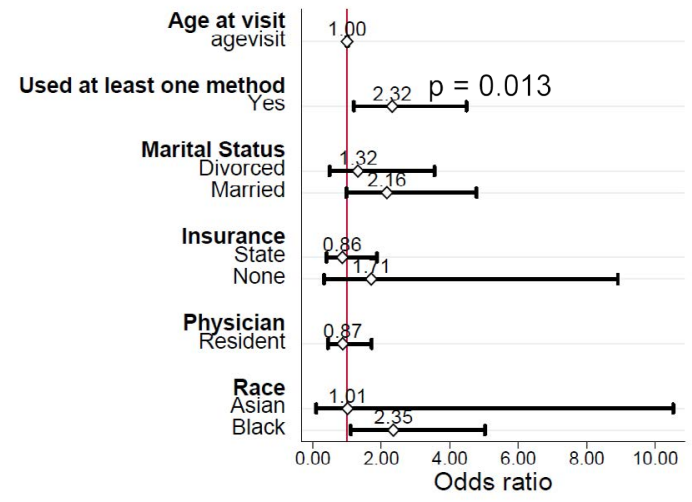

C

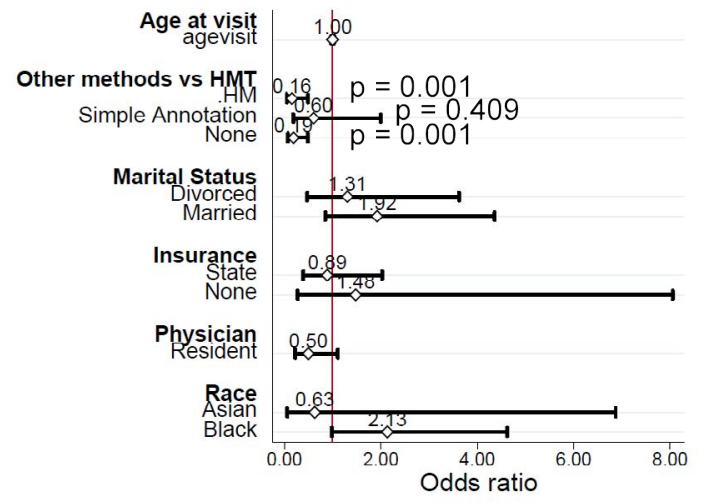

Figure 2: Results of Multiple Logistic Regression Analysis. a. HMT use was associated with 4.18 higher odds of completing cervical cancer screening than HMT non-use. b. The use of at least one reminder system was associated with double the odds of cervical cancer screening compared to not using any method of tracking preventive care. c. Controlling for age, marital status, insurance type, race, and physician level, .HM use was associated with $84 \%$ lower odds of cervical cancer screening compared to HMT use. Not using any reminder systems was associated with $81 \%$ lower odds of screening.

Consistent with previous literature, we found significantly higher odds of cervical cancer screening among married women compared to single women $[7,19]$. However, we did not find a significant association between insurance status and odds of cervical cancer screening. Possible explanations for this discrepancy include the small size of uninsured women in our sample as well as institutional coverage of preventive care services for uninsured patients in our study, considering that UF serves as a safety net provider for underserved populations. Another unexpected finding, was the significantly higher odds of screening among black/African American women compared to white/Caucasian participants. While not statistically significant, screening rates were lower in Asian women; consistent with previous studies [4]. Another statistically non-significant discrepancy was the higher rates of cervical cancer screening in Hispanic/Latina patients, which might also be explained by the small number of women who reported Hispanic/ Latina ethnicity.

The HMT is an innovative tool because it can easily be adapted and incorporated into paper-based medical charts or EMRs. In contrast to previously described reminder systems, the HMT allows providers to review their patient's preventive care at the appropriate time when they are ready to do so during the visit and to implement it where they would like within their documentation. The HMT can also be modified by each provider to fit the specific needs of any patient in addition to summarize the results and dates for all evidence-based preventive care services. Since it is easy to review and update at each visit, it minimizes the challenge of "fragmentation of health care information" and diminishes the time spent typing out paragraphs about all preventive care services [10]. Other advantages of the HMT are that it is simple, intuitive, and medical provider-friendly.

\section{Limitations}

Potential limitations of our study include possibly missing results for procedures performed outside of UF. However, we do not believe that one group of patients was more likely to have missing values compared to others. Since providers were not randomly assigned to HMT or non-HMT, our results might reflect intrinsic characteristics of physicians who choose to utilize HMTs. This limitation could be overcome in the future by randomly assigning providers to a reminder system. Since this study was performed at one academic institution, our results might not be generalizable to other institutions or settings. Future research is warranted to assess the effectiveness of HMT implementation in different healthcare settings, on other preventive care measures, and on patient outcomes.

\section{Conclusion}

Cervical cancer screening allows for cancer prevention and early detection, yet significant gaps remain in routine screening. Improving evidence-based cervical cancer screening represents an opportunity to reduce morbidity and mortality as well as associated health disparities. Physician recommendation is associated with higher completion of cancer screening [20,21]. In our study, HMT utilization by PCPs was 
associated with higher odds of cervical cancer screening, suggesting that this tool may provide a sustainable model for improving rates of evidence-based cervical cancer screening.

\section{References}

1. Siegel RL, Miller KD, Jemal A (2016) Cancer statistics 2016. CA Cancer J Clin 2016; 66: 7-30. doi: 10.3322/caac.21332. [Crossref]

2. Smith RA, Manassaram-Baptiste D, Brooks D, Cokkinides V, Doroshenk M, et al. (2014) Cancer screening in the United States, 2014: a review of current American Cancer Society guidelines and current issues in cancer screening. CA Cancer J Clin 64: 30-51. [Crossref]

3. National Center for Health Statistics (2015) Health, United States, 2014: With Special Feature on Adults Aged 55-64. Hyattsville, MD. [Crossref]

4. Smith RA, Andrews K, Brooks D, DeSantis CE, Fedewa SA, et al. (2016) Cancer screening in the United States, 2016: A review of current American Cancer Society guidelines and current issues in cancer screening. CA Cancer J Clin 66: 96-114. [Crossref]

5. Janerich DT, Hadjimichael O, Schwartz PE, Lowell DM, Meigs JW, et al. (1995) The screening histories of women with invasive cervical cancer, Connecticut. Am J Public Health 85: 791-794. [Crossref]

6. Moyer VA (2012) U.S. Preventive Services Task Force. Screening for cervical cancer: U.S. Preventive Services Task Force recommendation statement. Ann Intern Med 156: 880-891. [Crossref]

7. Limmer K, LoBiondo-Wood G, Dains J (2014) Predictors of cervical cancer screening adherence in the United States: a systematic review. J Adv Pract Oncol 5: 31-41. [Crossref]

8. Hitzeman N1, Xavier EM (2012) Interventions to increase cervical cancer screening rates. Am Fam Physician 85: 443-445. [Crossref]

9. Albrow R, Blomberg K, Kitchener H, Brabin L, Patnick J, et al. (2014) Interventions to improve cervical cancer screening uptake amongst young women: a systematic review. Acta Oncol 53: 445-451. [Crossref]

10. Dexheimer JW, Talbot TR, Sanders DL, Rosenbloom ST, Aronsky D (2008) Prompting clinicians about preventive care measures: a systematic review of randomized controlled trials. J Am Med Inform Assoc 15: 311-320. [Crossref]
11. Garg AX, Adhikari NK, McDonald H, Rosas-Arellano MP, Devereaux PJ, et al. (2005) Effects of computerized clinical decision support systems on practitioner performance and patient outcomes: a systematic review. JAMA 293: 1223-1238. [Crossref]

12. Shea S, DuMouchel W, Bahamonde L (1996) A meta-analysis of 16 randomized controlled trials to evaluate computer-based clinical reminder systems for preventive care in the ambulatory setting. J Am Med Inform Assoc 3: 399-409. [Crossref]

13. Kaushal R, Shojania KG, Bates DW (2003) Effects of computerized physician order entry and clinical decision support systems on medication safety: a systematic review. Arch Intern Med 163: 1409-1416. [Crossref]

14. Romano MJ, Stafford RS (2011) Electronic health records and clinical decision support systems: impact on national ambulatory care quality. Arch Intern Med 171: 897-903. [Crossref]

15. Luu SW, Sattari M (2016) Improving influenza vaccination rate with health maintenance tables. Abstracts from the 2016 Society of General Internal Medicine Annual Meeting. J Gen Intern Med 31: S271-S272.

16. STATA (for Windows) [computer program] (2015) Version 14. College Station, TX StataCorp LP.

17. Sittig DF, Krall MA, Dykstra RH, Russell A, Chin HL (2006) A survey of factors affecting clinician acceptance of clinical decision support. BMC Med Inform Decis Mak 6: 6. [Crossref]

18. Bright TJ, Wong A, Dhurjati R, Bristow E, Bastian L, et al. (2012) Effect of clinical decision-support systems: a systematic review. Ann Intern Med 157: 29-43. [Crossref]

19. Hanske J, Meyer CP, Sammon JD, Choueiri TK, Menon M, et al. (2016) The influence of marital status on the use of breast, cervical, and colorectal cancer screening. Prev Med 89: 140-145. [Crossref]

20. Sabatino SA, Lawrence B, Elder R, Mercer SL, Wilson KM, et al. (2012) Community Preventive Services Task Force. Effectiveness of interventions to increase screening for breast, cervical, and colorectal cancers: nine updated systematic reviews for the guide to community preventive services. Am J Prev Med 43: 97-118. [Crossref]

21. Kern LM, Barrón Y, Dhopeshwarkar RV, Edwards A, Kaushal R (2013) HITEC Investigators. Electronic health records and ambulatory quality of care. J Gen Intern Med 28: 496-503. [Crossref]

Copyright: (C2018 Romero de Mello Sa SA. This is an open-access article distributed under the terms of the Creative Commons Attribution License, which permits unrestricted use, distribution, and reproduction in any medium, provided the original author and source are credited. 